\title{
A Min-Max SCHEduling LoAd BaLANCED APPROACH TO ENHANCE ENERGY EFFICIENCY AND PERFormanCE OF MOBILE ADHOC NETWORKS
}

\author{
K.Venkatachalapathy ${ }^{1}$ and D.Sundaranarayana ${ }^{2}$ \\ ${ }^{1}$ Research Scholar, Department of Computer Science and Engineering, \\ Annamalai University, Chidambaram, India \\ ${ }^{2}$ Professors, Department of Computer and Information Science, \\ Annamalai University, Chidambaram, India
}

\begin{abstract}
Energy efficiency and traffic management in Mobile Ad hoc Networks (MANETs) is a complex process due to the self-organizing nature of the nodes. Quality of service (QoS) of the network is achieved by addressing the issues concerned with load handling and energy conservation. This manuscript proposes a min-max scheduling (M2S) algorithm for energy efficiency and load balancing (LB) in MANETs. The algorithm operates in two phases: neighbor selection and load balancing. In state selection, the transmission of the node is altered based on its energy and packet delivery factor. In the load balancing phase, the selected nodes are induced by queuing and scheduling the process to improve the rate of load dissemination. The different processes are intended to improve the packet delivery factor (PDF) by selecting appropriate node transmission states. The transmission states of the nodes are classified through periodic remaining energy update; the queuing and scheduling process is dynamically adjusted with energy consideration. A weight-based normalized function eases neighbor selection by determining the most precise neighbor that satisfies transmission and energy constraints. The results of the proposed M2SLB (Min-Max Scheduling Load Balancing) proves the consistency of the proposed algorithm by improving the network throughput, packet delivery ratio and minimizing delay and packet loss by retaining higher remaining energy.
\end{abstract}

\section{KEYWORDS}

Energy Efficiency, Load Balancing, MANET, Packet Delivery Factor, Queuing and Scheduling.

\section{INTRODUCTION}

Mobile ad hoc network (MANET) is a collection of autonomous self-organizing terminal nodes interconnected through wireless links. The terminal nodes exhibit two types of behavior: host and router for sending, receiving and forwarding data. Data handling and transmitting is expedited by selecting appropriate routing protocols. The nodes are mobile in nature, capable of moving around the network with a varying speed; the network is volatile. This dynamic network does not require any special infrastructure and is flexible for communication with any neighbor nodes. The nodes communicate through radio units and they rely on their neighbors for transmitting data over a long distance in a multi-hop fashion [1]. Routing protocols are designed to cope-up with the intrinsic nature of the nodes to achieve better network performance. However, quality-of-service (QoS) is still a demanding task that cannot be served by the conventional routing protocols. QoS fulfillment varies with the demands of the end-users that are also controlled by the nature of the nodes. Conventional routing protocols and algorithms designed so far concentrates in minimizing network traffic or reducing delay due to multi-hops. This result in partial achievement of network QoS, for which ongoing research process serves different solutions [2]. 
The nodes are powered by built-in batteries that drain with time. The nodes exploit battery power for sending and receiving data. The neighboring nodes that forward data also drain energy other than the source and destination. This increases the energy expenses of MANET that needs to be controlled for achieving efficiency. There are multiple factors affecting energy efficiency, the resource constraint nature of the nodes is the prominent factor. Implementing an efficient energy conservation policy for the mobile nodes will improve the network lifetime by confining the rate of energy exploitation. The nodes are deployed in hostile environments where replacing and recharging of the battery is practically impossible. Contrarily, the node has to dispatch all of its communication within the dead state to ensure network reliability. The aforementioned efficiency in the network is achieved by overseeing the protocols that are energy proficient which is typically designed for this purpose. Similarly, complex optimization methods increase the communication cost of the network by imposing a task load on the nodes. The design goal of energy efficient routing algorithms and protocol is to improve network lifetime by minimizing unnecessary energy exploitation and to prevent earlier energy drain of the nodes [3, 4].

Traffic management is another issue in the mobile network to handle congestion. QoS of the network solely relies on the incoming the network traffic that is to be rationalized to prevent congestion at nodes and communication links. Load balancing is the conventional solution to handle network traffic and to prevent information overloading. Load balancing utilizes all the resources in the network in accordance with the routing protocols to deliver information to appropriate destinations. Along with energy efficiency, routing protocols are intended to improve load dissemination through the available intermediate nodes. As the nodes possess limited storage, data overloading cannot be prevented, resulting in bottlenecks. Appropriate load balancing schemes are tested with wireless networks to minimize the rate of congestion through uniform and on-demand load distribution. Avoiding unnecessary energy exploitation and earlier energy drain are some of the joint benefits of load balancing [5-7].

\section{RELATED WORKS}

Farooq Aftab et al. [8] proposed a self-organizing clustering scheme for improving network scalability and endurance. The authors address network congestion issues by varying cluster sizes dynamically. Further improvements in the network such as energy efficiency, cluster head changes are optimized with the consideration of node energy and mobility.

Constructive-Relay-based cooperative routing (CRCPR) protocol is introduced by Jingwen Bai et al. [9] to improve the communication rates of MANET nodes. This protocol considers the energy utilization and conservation rates of the nodes and link failure probability to determine the optimal routing paths to the destination. This protocol improves network throughput with prolonged network life span.

Ad hoc on demand multipath distance vector with fitness function (FF-AOMDV) [10] is an approach for improving the performance and the efficiency of energy usage in the mobile ad-hoc network environment. The fitness of the route is evaluated by considering the energy and distance of the neighboring nodes. Conventional multipath routing is deviated from utilizing its distance estimation to prolong the network lifetime.

Dynamic hop-aware buffering (DHAB) propose by Al-Mahdi and Kalil [11] is designed for minimizing packet loss and delay in MANETs. Based on the packet's priority, the buffer of the nodes is virtually allocated for transmission over long hops. The buffering technique is designed to dynamically adjust with the arriving traffic using a Markov chain model. 
Magán-Carrión et al. [12] introduced a dynamic relay placement solution for improving the operational performance of MANETs. A combination of particle swarm optimization (PSO) and model predictive control (MPC) is used for the above- mentioned placement and selection. This bi-objective optimization improves network throughput and link connectivity jointly.

Energy efficient lifetime aware multicast (EELAM) [13] is exclusively designed for MANETs for improving its route discovery efficiency. EELAM is a multicast routing process that employs a genetic algorithm for selecting nodes based on residual energy and energy exploitation. The fitness function of the genetic process is intended to improve the energy efficiency of the network.

Dynamic multi-stage tandem queue modeling-based congestion adaptive routing (DTQCAR) [14] is introduced to improve the packet delivery ratio of the network besides unplanned network traffic. This adaptive routing identifies congestion, alerts the neighbors for handling network traffic for minimizing packet loss. This congestion control routing identifies congestion-free neighbors for disseminating load.

The Power and load aware (PLA) multipath routing scheme are proposed by Ali et al. [15] for prolonging MANET lifetime despite varying network load. This multipath routing is constructed based on dynamic source routing protocol (DSR) and the protocol identifies neighbors using cost function. With this cost function, the source identifies multiple disjoint optimal paths to the destinations.

Learning automata (LA) based route selection process is introduced in [16] for improving the service quality of MANETs. The feedback mechanism of LA helps the source node to discover optimal routes satisfying energy, link stability, and delay factors. This method is more apt for a network with varying node density and velocity.

\section{PROPOSED METHOdOLOGY}

The proposed Min-Max Scheduling load balancing algorithm (M2SLB) is designed to improve energy efficiency and load balancing in mobile nodes. Different from the existing approaches, this algorithm considers two definitions with min-max constraints along with the energy of the nodes before scheduling. The scheduling process is dynamic to cope-up with the mobility of the nodes where a distinct set of evaluation criterion is considered. The min-max is formulated for two different states of the nodes: energy retained state and half drain state. Link expiration time (LET) and packet delivery factor (PDF) is the other two duos for the states of the nodes to improve load handling. The different combinations of node factors aid to improve network performance by improving throughput, packet delivery ratio, with lesser delay and energy exploitation.

\subsection{Network Model}

We represent a MANET in the form of a graph $\mathrm{G}=(\mathrm{N}, \mathrm{V})$ where, $\mathrm{N}$ is the set of mobile nodes that are connected through a set of vertices $V$. Consider two nodes $n_{i}$ and $n_{j}$, the nodes are said to be direct neighbors if distance $\mathrm{d}\left(\mathrm{n}_{\mathrm{i}}, \mathrm{n}_{\mathrm{j}}\right)<R(i \| j)$, where $\mathrm{R}$ is the communication range of the mobile node. 


\subsubsection{Energy Model}

Mobile nodes utilize energy for transmitting and receiving packets. Each node is fitted with a battery source with initial energy $E_{\text {init. }}$. The energy utilization of a node $E_{u}$ is the summation of transmitting $\left(\mathrm{E}_{\mathrm{tm}}\right)$ and receiving $\left(\mathrm{E}_{\mathrm{rm}}\right)$ energies that is represented in equation (1)

$$
\mathrm{E}_{\mathrm{u}}=\mathrm{E}_{\mathrm{tm}}+\mathrm{E}_{\mathrm{rm}}
$$

With the knowledge of energy utilization, the remaining energy $\left(\mathrm{E}_{\text {rem }}\right)$ of the node is estimated as

$$
\mathrm{E}_{\mathrm{rem}}=\mathrm{E}_{\text {init }}-\mathrm{E}_{\mathrm{u}}
$$

\subsection{Min-Max Scheduling for Energy Efficient Load Balancing (M2SLB) Algorithm}

The methodology is modeled to handle two different situations before scheduling; before half drain and after half drain of the node. The half drain $\left(\mathrm{E}_{\mathrm{h}}\right)$ of a node is defined as the point at which its remaining energy reaches exactly half of its initial energy. We notify the fore and after drain states as active transmission and light transmission correspondingly. In active and light transmission states, the neighbor selection is prioritized based on energy for ensuring the energy efficiency of the network. The entire process of M2SLB is divided as neighbor selection and load distribution. The Neighbor selection phase is responsible for conserving energy and load distribution improves the transmission rate of the network.
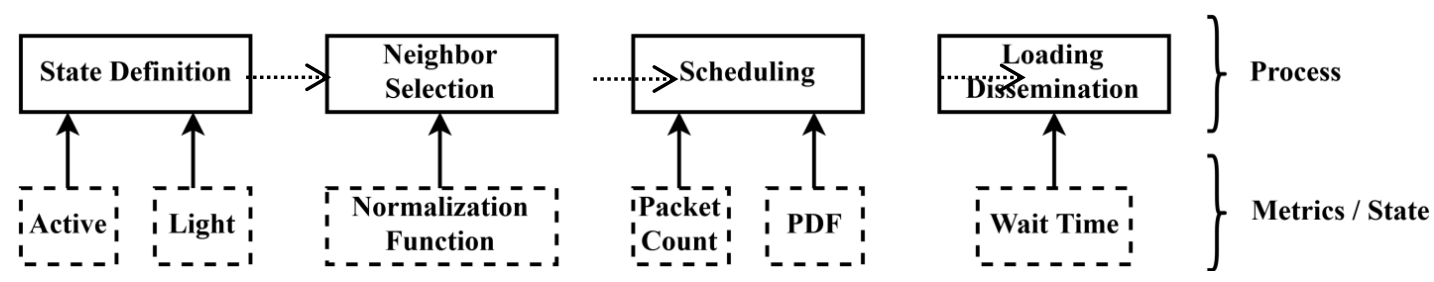

Figure 1. Process of M2SLB

We consider a normalization function $(\mathrm{f}(\mathrm{N}))$ to ease neighbor selection, from which energy retaining nodes are selected for transmission. The distinct metrics are balanced by assigning appropriate weights for the considered metrics depending upon the state classification. Figure 1 illustrates the process of M2SLB with their properties and functionality considerations in each stage.

\subsubsection{State Definition based neighbor selection}

The state definition of a node is classified as active or light transmission. This classification is based on the remaining energy of the node. If a node $\mathrm{E}_{\text {rem }}$ is less than its half drain then, the node is in light transmission state otherwise it is inactive transmission state. During the active transmission period, the neighbor selection is facilitated by evaluating $\mathrm{E}_{\mathrm{tm}}$ and link expiry time (LET). LET indicates the lifetime of the vertex $v \in V$, i.e. the link between two nodes. The LET of a vertex between $n_{i}$ and $n_{j}$ is estimated using equation (3). 


$$
\operatorname{LET}_{\mathrm{n}_{\mathrm{i}}-\mathrm{n}_{\mathrm{j}}}=\frac{-(\mathrm{ab}+\mathrm{cd})+\sqrt{\left(\mathrm{a}^{2}+\mathrm{c}^{2}\right) \mathrm{R}^{2}-(\mathrm{ad}-\mathrm{bc})^{2}}}{\mathrm{a}^{2}+\mathrm{c}^{2}}
$$

Where $\mathrm{a}=\mathrm{N}_{\mathrm{n}_{\mathrm{i}}} \cos \theta_{\mathrm{n}_{\mathrm{i}}}-\mathrm{V}_{\mathrm{n}_{\mathrm{j}}} \cos \theta_{\mathrm{n}_{\mathrm{j}}}, \mathrm{b}=\mathrm{x}_{\mathrm{n}_{\mathrm{i}}}-\mathrm{x}_{\mathrm{n}_{\mathrm{j}}}, \mathrm{c}=\mathrm{V}_{\mathrm{n}_{\mathrm{i}}} \sin \theta_{\mathrm{n}_{\mathrm{i}}}-\mathrm{V}_{\mathrm{n}_{\mathrm{j}}} \sin \theta_{\mathrm{n}_{\mathrm{j}}}$, $\mathrm{d}=\mathrm{y}_{\mathrm{n}_{\mathrm{i}}}-\mathrm{y}_{\mathrm{n}_{\mathrm{j}}}$

The other variables used are $\left(\mathrm{x}_{\mathrm{n}_{\mathrm{i}}}, \mathrm{y}_{\mathrm{n}_{\mathrm{i}}}\right)$ and $\left(\mathrm{x}_{\mathrm{n}_{\mathrm{j}}}, \mathrm{y}_{\mathrm{n}_{\mathrm{j}}}\right)$ represents the coordinates of the nodes $\mathrm{n}_{\mathrm{i}}$ and $n_{j}$ moving with a velocity of $V_{n_{i}}$ and $V_{n_{j}}$ respectively. The nodes are mobile with a mobility deviation of $\theta_{n_{i}}$ and $\theta_{n_{j}}$ correspondingly with the estimation of LET, the normalization function is estimated with the weight $w_{1}$ and $w_{2}$ as given in the below equation (4)

$$
\mathrm{f}(\mathrm{N})=\mathrm{w}_{1} * \mathrm{E}_{\mathrm{tm}}+\mathrm{w}_{2} * \mathrm{LET}
$$

Here, $\mathrm{E}_{\mathrm{tm}}$ must be minimum and LET is maximum, which is an ideal condition that is satisfied at random time. There are two cases wherein the node attributes affects the normalization function:

Case 1: The nodes possess higher mobility (i.e.) a neighboring node selected is induced under variable velocity. In this case, the weight of the LET metric is given a higher choice wherew $_{2}>\mathrm{w}_{1}$. This condition is valid until $\mathrm{E}_{\text {rem }}>\mathrm{E}_{\mathrm{h}}$

Case 2: The remaining energy of the node ceases more abruptly, in this case, $w_{1}>w_{2}$ and the node moves to light transmission state.

When a node moves to a light transmission state, the remaining energy and PDF are considered selection; the min-max is violated. The PDF at the destination is estimated using equation (5)

$$
\mathrm{PDF}=\prod_{\mathrm{i}=\mathrm{n}}^{\mathrm{n}+\mathrm{h}-1}\left(1-\mathrm{t}_{\mathrm{loss}}\right)
$$

Where $\mathrm{t}_{\text {loss }}$ the packets lost in the transmission, $\mathrm{n}$ represents the sender/ transmitting node and $\mathrm{h}$ is the hop distance between source and destination. Different from the above cases, weights are assigned with balanced values (i.e.) the weights are equally considered. Obviously, the link transmission rate in the specific vertex is reduced to cope- up with the available energy of the node. From either of the cases, $\max \left\{\mathrm{f}\left(\mathrm{N}_{1}\right), \mathrm{f}\left(\mathrm{N}_{2}\right), \ldots \mathrm{f}\left(\mathrm{N}_{\mathrm{j}}\right)\right\}$ is selected as the next neighbor for transmitting packets. The process of state definition based neighbor selection is illustrated in the below Figure 2.

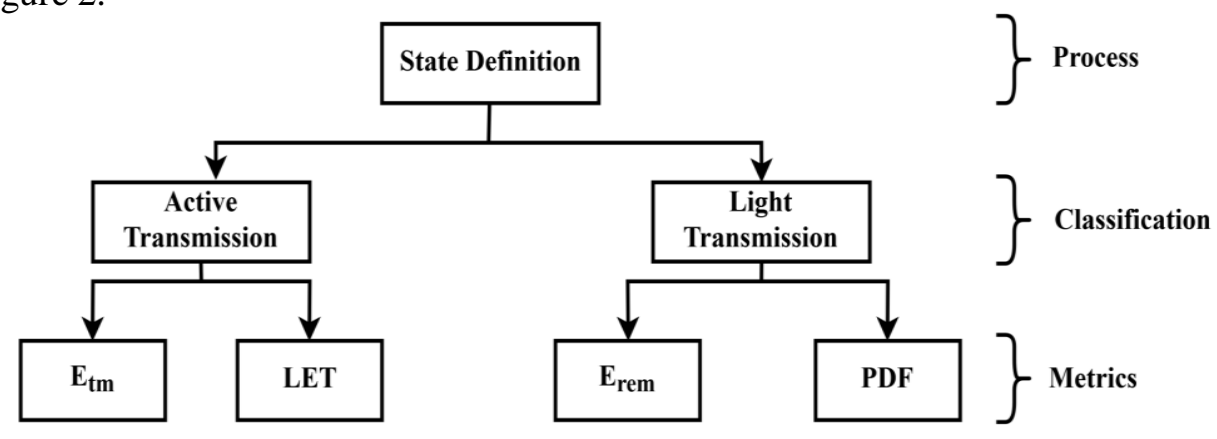

Figure 2. State Definition Process Illustrations 
The process of energy dependent neighbor selection is described in Algorithm 1.

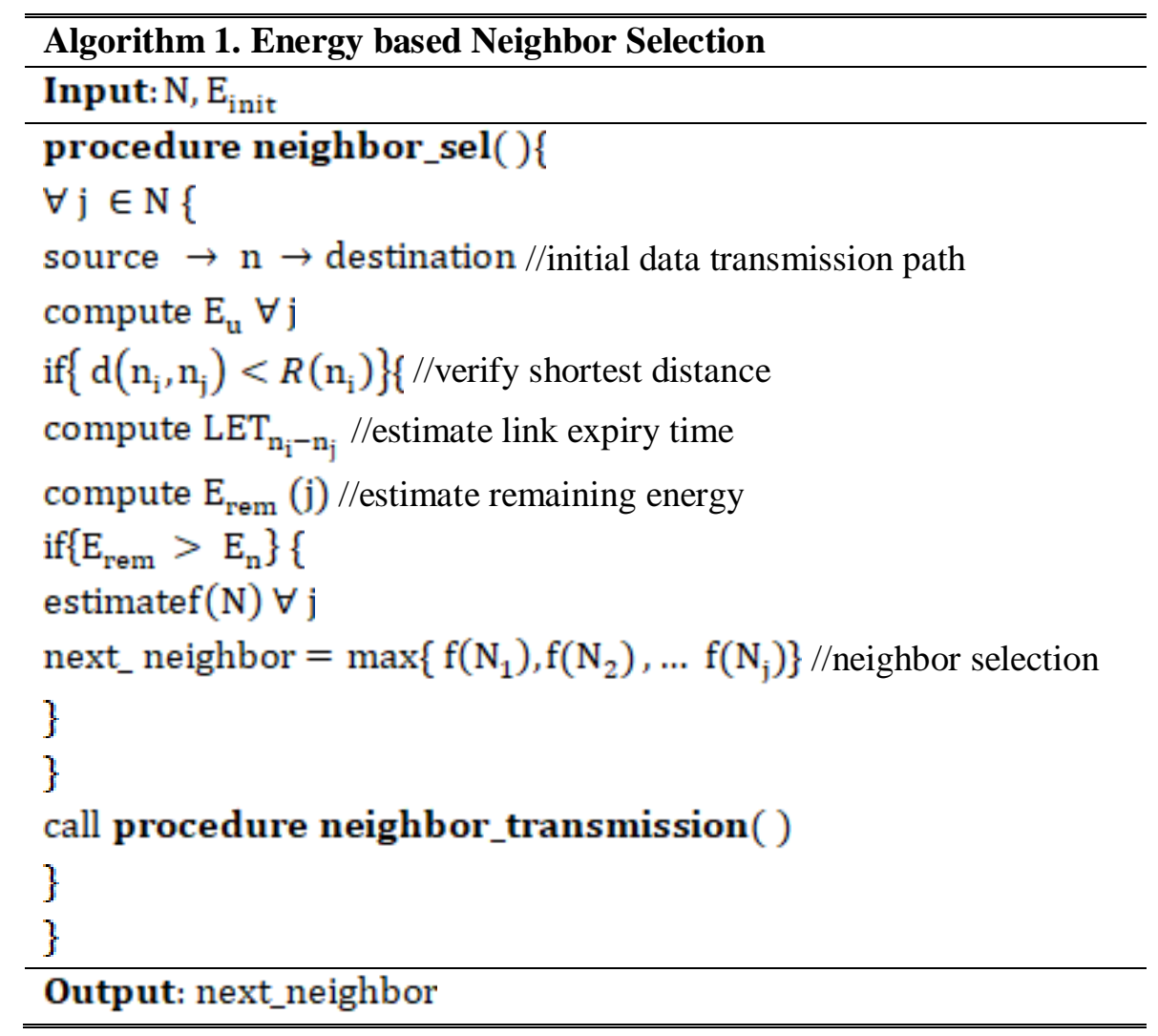

\subsubsection{Scheduling and Transmission for Load Balancing}

Figure 3 portrays the general representation of scheduling and transmission process.

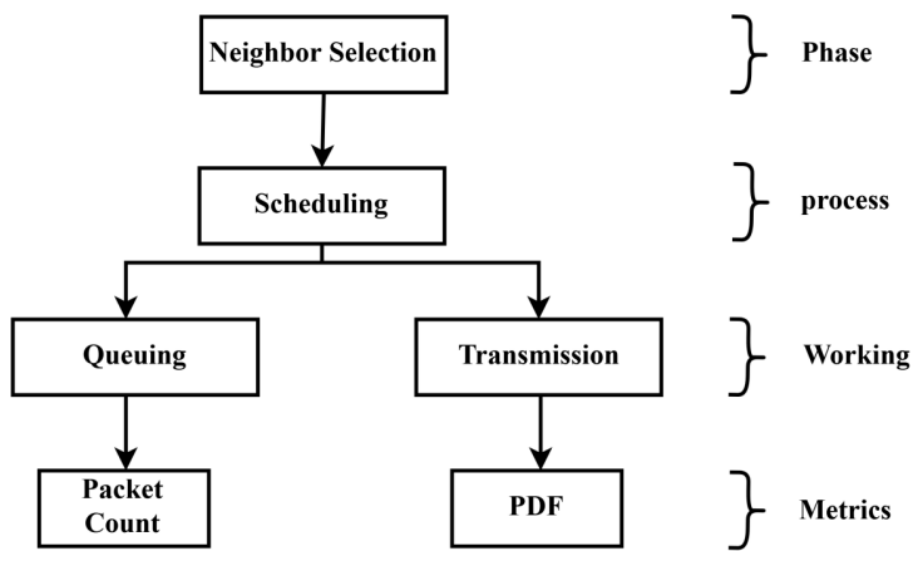

Figure 3. Scheduling and Transmission Process

In the scheduling process, first -in - first out method is followed to distribute load across the network. Before the scheduling process, the selected nodes are utilized to store and forward incoming traffic packets. The Queuing process is temporary to prevent packets being lost in congestion. The rate of queuing relies on the number of packets being transmits. The packets transmitted $\left(\mathrm{t}_{\mathrm{p}}\right)$ is then estimated as 
International Journal of Computer Networks \& Communications (IJCNC) Vol.11, No.3, May 2019

$$
t_{p}=\frac{d_{r}}{t_{t}}
$$

Where $d_{r}$ and $t_{t}$ are the data rate and transmitting time observed for a vertex V.

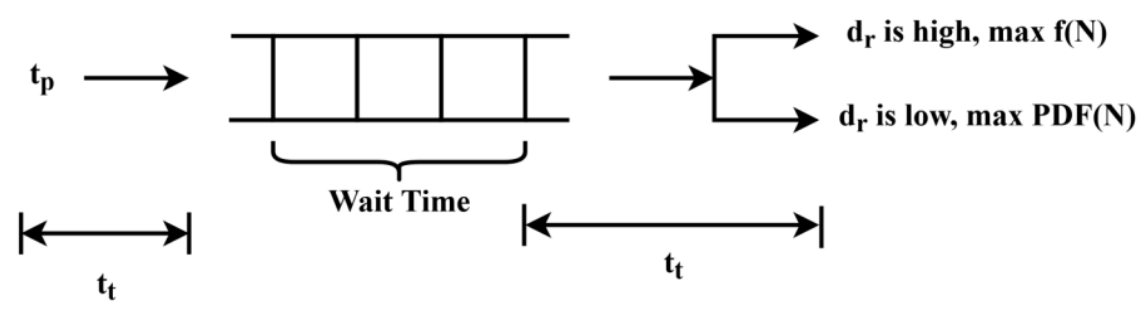

Figure 4. Queuing and Packet Dissemination

The queue of the selected neighbor is filled in the order of $t_{p}$ received. The accepted packets are forwarded with a small waiting time. The further neighbor set is selected by estimating PDF at the destination through that neighbor. For energy retained node, $\max \left\{\operatorname{PDF}\left(\mathrm{n}_{1}\right), \operatorname{PDF}\left(\mathrm{n}_{2}\right), \ldots \ldots . . . \mathrm{PDF}\left(\mathrm{n}_{\mathrm{j}}\right)\right\}$ is selected for further transmission. The above condition is optimal for case 1 discussed earlier. For nodes entering light transmission state, $t_{p}$ is less and hence wait time is less but PDF through the node must satisfy maximum delivery or maximum loss. Those nodes are prevented from being overloaded by avoiding queue overflow achievement. The process of PDF scheduling and packet queuing is illustrated in figure 4. Algorithm 2 describes the process of scheduling and transmission of neighbor selection.

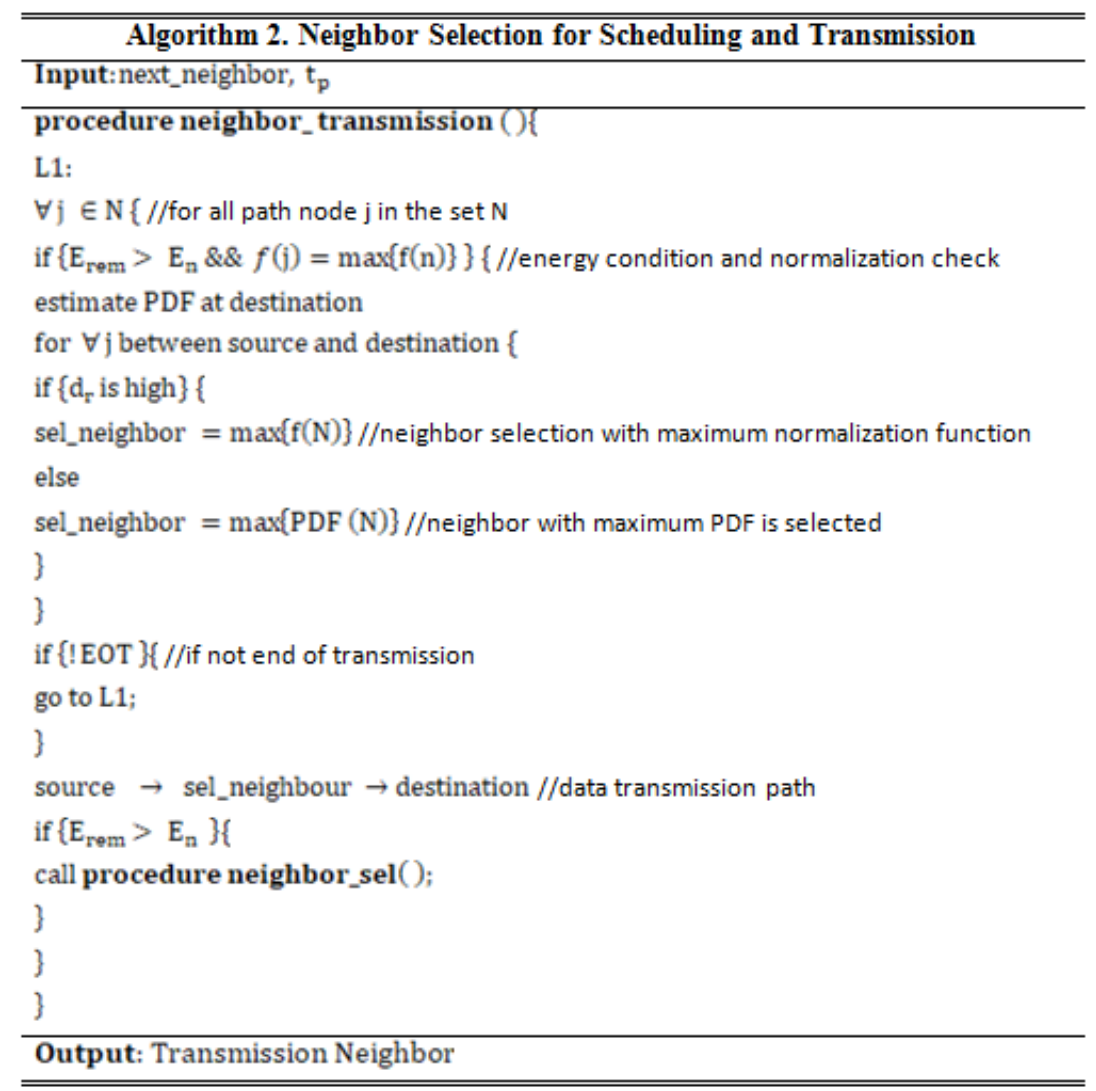




\section{RESULTS AND DISCUSSION}

The proposed min-max scheduling for energy efficient load balancing has been simulated in the Network simulator -2 simulation tool and its performance under different parameters are examined. A comparative analysis of the proposed method with FF-AOMDV [10] and DTQCAR [14] takes place for the metrics: throughput, packet delivery ratio, delay, remaining energy and packet loss. Table 1 summarizes the simulation parameters and their values.

Table 1. Simulation Parameters and Values

\begin{tabular}{ll}
\hline \hline Parameter & Value \\
\hline Network Dimension & $1000 \mathrm{~m} \times 1000 \mathrm{~m}$ \\
Mobile Nodes & 50 \\
MAC & 802.11 \\
Range of the Node & $250 \mathrm{~m}$ \\
Application Type & Constant Bit Rate \\
$\mathrm{E}_{\text {init }}$ & $20 \mathrm{~J}$ \\
Pause Time & $2 \mathrm{~ms}$ \\
Queuing Model & FIFO \\
\hline \hline
\end{tabular}

\subsection{Throughput Analysis}

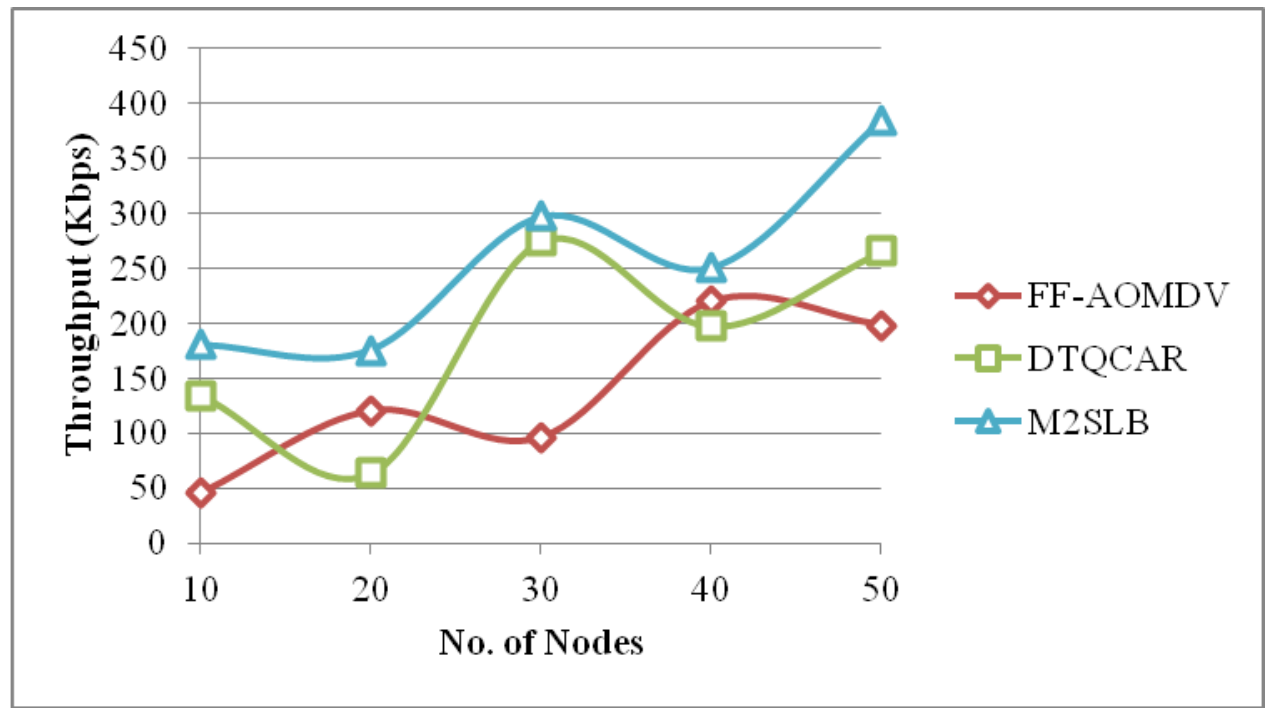

Figure 5. Throughput Comparisons

Figure 5 illustrates the comparisons of throughput observed in the proposed and existing methods. In the proposed M2SLB, the incoming network traffic is disseminated through dynamic queuing and adjustable link rates. The number of stagnant packets in the node queue is less, as the further neighbors and transmission rates are decided upon the PDF and $\mathrm{f}(\mathrm{N})$ factors. With the varying data rate, node transmission is modeled to support both load handling and energy awareness. This feature improves the throughput, where the throughput of the existing DTQCAR and FFAOMDV is $31.11 \%$ and $48.48 \%$ lower when compared to the proposed M2SLB (at the number of nodes $=50$ ). 


\subsection{Packet Delivery Ratio Analysis}

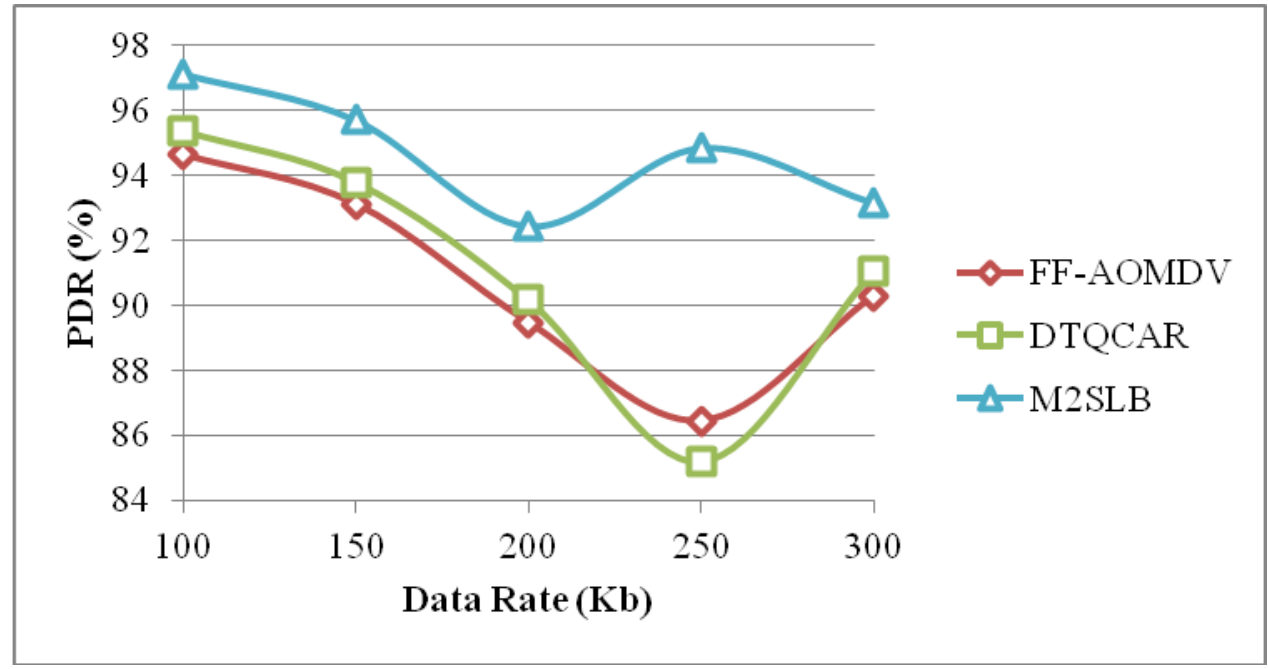

Figure 6. Packet Delivery Ratio (PDR) Comparisons

The diminishing factors in the network due to network load and energy are addressed by selecting neighbors that achieve higher PDF. In both energy efficient neighbor selection and queuing process, the transmission is preceded by selecting neighbors ensuring maximum PDF. This factor is achieved for varying data rates; the energy of the nodes is also accounted for ensuring seamless transmission. In both active and light transmission, the neighbors satisfy max PDF to achieve higher delivery ratio (Figure 6). The proposed Packet delivery ratio has been improved, where the existing DTQCAR and FF- AOMDV has $2.25 \%$ and $3.07 \%$ (at data rate $=300 \mathrm{~Kb}$ ) lower PDR than M2SLB.

\subsection{Delay Analysis}

The comparisons of delays between the existing and proposed methods are illustrated in Figure 7. The number of packet loss in the proposed M2SLB is less for which the retransmission required is less. The waiting time of the packets is less as the neighbor in both states is instantly identified to forward packets to the destination. Therefore, along with the round trip time of the packet, less waiting time is accounted.

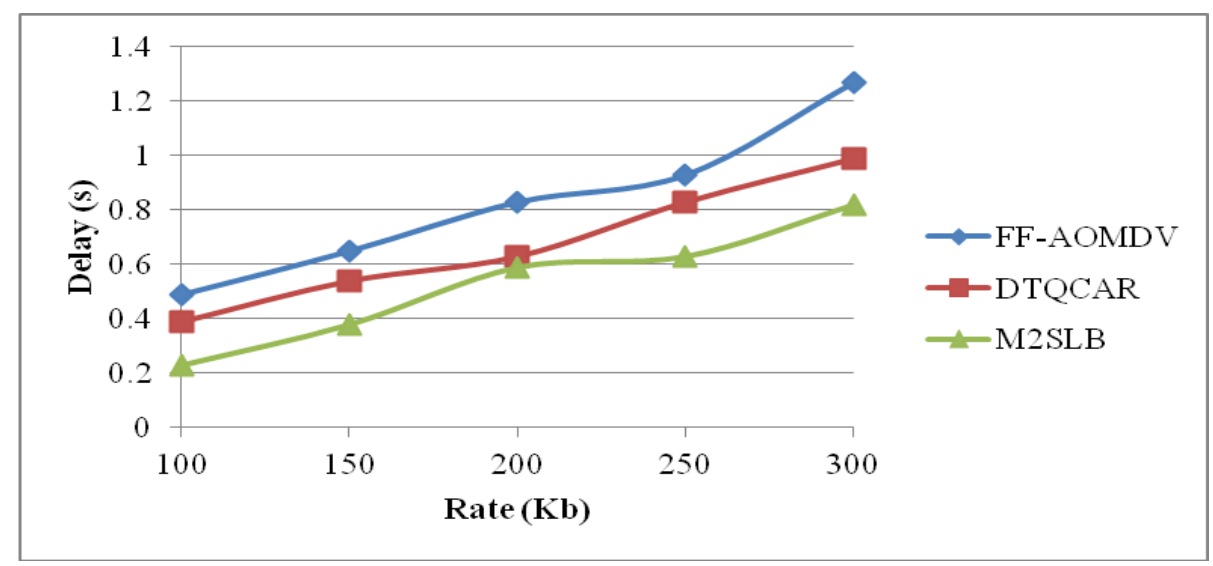

Figure 7. Comparisons of Delays 
This time is comparatively less as the retransmission is controlled. The proposed M2SLB minimizes delay by $17.17 \%$ and $35.43 \%$ compared to DTQCAR and FF-AOMDV respectively $($ Rate $=300 \mathrm{~Kb})$.

\subsection{Remaining Energy}

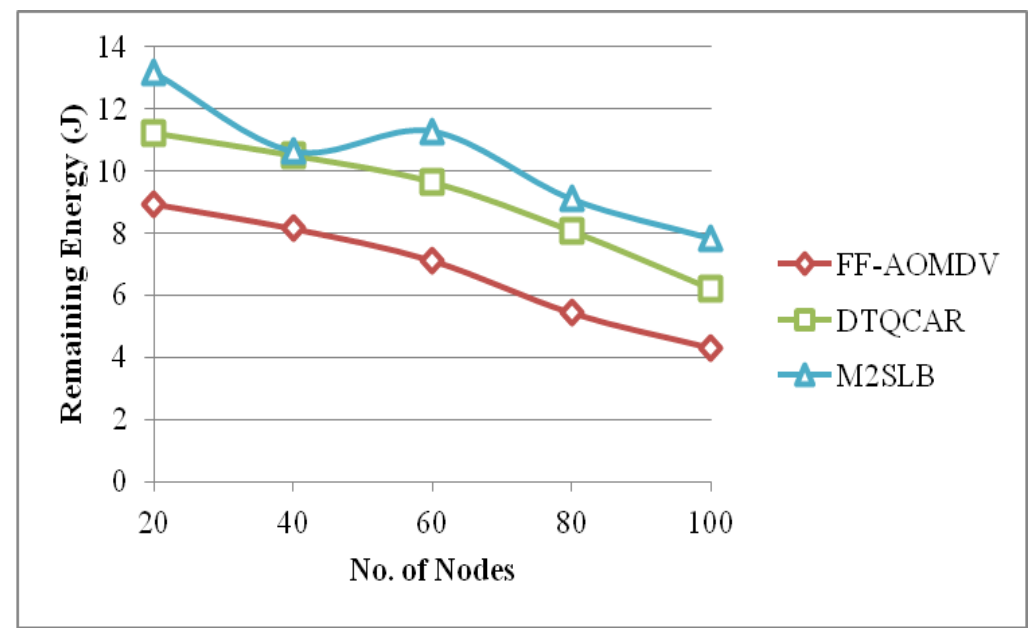

Figure 8. Remaining Energy Analysis Comparisons

In the state definition process of M2SLB, the transmission is classified based on the remaining energy of the nodes. On estimating the remaining energy, the transmission and queuing rates of the nodes are dynamically adjusted to improve the flow of $t_{p}$. Unlike the other methods, the lesser enduring energy nodes are prevented from handling higher traffic to conserve their lifetime. The nodes are prevented from draining all of its energy, by distributing the load among the available neighbors. Both the energy and load handling features of the proposed method aids to retain higher remaining energy when compared with previous DTQCAR and FF-AOMDV, where they have $20.94 \%$ and $45.08 \%$ lower remaining energy respectively when compared with M2SLB when the number of nodes $=100$.

\subsection{Packet Loss Analysis}

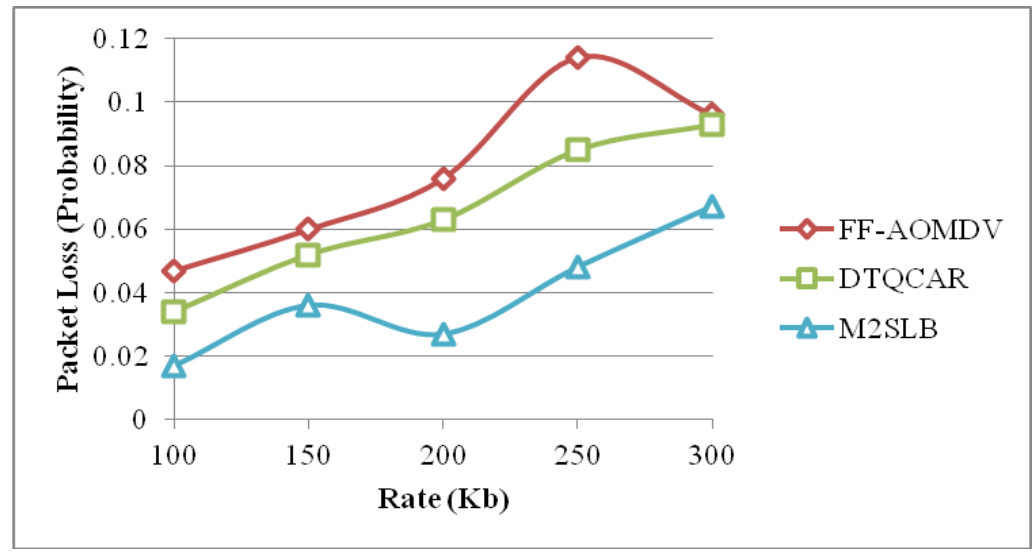

Figure 9. Packet Loss Factor Comparisons 
Figure 9 shows the packet loss factor comparisons of the proposed M2SLB with the existing methods. Here, as the comparison is verified a factor, the maximum value of the y-axis is 1 . Packet loss which is caused by energy and traffic congestion is resolved by selecting energy efficient and delivery assisting nodes in the network. The Initial state segregation process and later queuing and scheduling process improve the transmission rate with controlled delay. Henceforth, the proposed M2SLB minimizes packet loss by $27.95 \%$ and $30.20 \%$ at the time of congestion and queuing compared to the existing DTQCAR and FF-AOMDV when the rate is $300 \mathrm{~Kb}$. Table 2 presents the comparative values of the simulation for the above Parameters.

Table 2. Comparative tabulation of Results

\begin{tabular}{llll}
\hline \hline Metric & FF-AOMDV & DTQCAR & M2SLB \\
\hline Throughput (Kbps) & 198.25 & 265.09 & 384.82 \\
PDR (\%) & 90.28 & 91.04 & 93.14 \\
Delay (s) & 1.27 & 0.99 & 0.82 \\
Remaining Energy (J) & 4.3 & 6.19 & 7.83 \\
Packet Loss Probability & 0.096 & 0.093 & 0.067 \\
\hline \hline
\end{tabular}

\section{CONCLUSiON}

This manuscript introduces a min-max scheduling (M2S) algorithm for achieving energy efficiency and load balancing in MANETs. This algorithm accounts the energy based link stability for improving the energy efficiency of the network. Based on the energy, the nodes are classified under two stages that aids load distribution ensuring higher packet delivery. In the load balancing phase, the selected nodes are influenced by the queuing and scheduling process to improve the rate of data handling irrespective of the type of traffic. From the experimental results, it is seen that the proposed algorithm, on average, improves throughput and PDR by $39.82 \%$ and $2.66 \%$ correspondingly by retaining $33.01 \%$ higher remaining energy, where minimizing delay and packet loss by $26.3 \%$ and $29.07 \%$. The experimental results prove the consistency of the algorithm by improving network performance and energy efficiency in terms of throughput, packet delivery, delay, packet loss and remaining energy.

\section{REFERENCES}

[1] T. Spyropoulos, R. N. B. Rais, T. Turletti, K. Obraczka, and A. Vasilakos, "Routing for disruption tolerant networks: taxonomy and design," Wireless Networks, vol. 16, no. 8, pp. 2349-2370, Oct. 2010 .

[2] S. Batabyal and P. Bhaumik, "Mobility Models, Traces and Impact of Mobility on Opportunistic Routing Algorithms: A Survey," IEEE Communications Surveys \& Tutorials, vol. 17, no. 3, pp. 1679-1707, 2015.

[3] K.Venkatachalapathy, D.Sundaranarayana, "A Survey on Energy Efficiency Issues and solutions in Mobile ad-hoc Networks," International Journal of advanced computational engineering and networking, vol. 5, no. 9, pp. 38-44, 2017.

[4] D.Sundaranarayana, K.Venkatachalapathy, "An Effectual load distribution approach based on transmission power and topology controlled clustered environment in mobile adhoc network," International Journal of Computer Applications, vol.179, no. 25, pp. 34-38, 2018. 
International Journal of Computer Networks \& Communications (IJCNC) Vol.11, No.3, May 2019

[5] K.Venkatachalapathy, D.Sundaranarayana, "Energy Efficient modified butterfly optimization based clustering algorithm for cluster head selection in mobile ad hoc network," Journal of advanced research in dynamical and control systems, vol.2, Special isssue, pp.1521-1531, 2018.

[6] Mohammed Abdul waheed, K.Karibasappa, "QOS Routing for Heterogeneous MANET's," International journal of computer engineering sciences (IJCES), vol.2, Issue.3, pp.77-81, 2012.

[7] P. F. A. Selvi and M. M.s.k, "Ant Based Multipath Backbone Routing for Load Balancing in MANET," IET Communications, 2016.

[8] F. Aftab, Z. Zhang, and A. Ahmad, "Self-Organization Based Clustering in MANETs Using Zone Based Group Mobility,” IEEE Access, vol. 5, pp. 27464-27476, 2017.

[9] J. Bai, Y. Sun, C. Phillips, and Y. Cao, "Toward Constructive Relay-Based Cooperative Routing in MANETs,” IEEE Systems Journal, vol. 12, no. 2, pp. 1743-1754, 2018.

[10] A. Taha, R. Alsaqour, M. Uddin, M. Abdelhaq, and T. Saba, "Energy Efficient Multipath Routing Protocol for Mobile Ad-Hoc Network Using the Fitness Function,” IEEE Access, vol. 5, pp. 1036910381, 2017.

[11] H. Al-Mahdi and M. A. Kalil, “A Dynamic Hop-Aware Buffer Management Scheme for Multi-hop Ad Hoc Networks,” IEEE Wireless Communications Letters, pp. 1-1, 2016.

[12] R. Magán-Carrión, J. Camacho, P. García-Teodoro, E. F. Flushing, and G. A. D. Caro, “A Dynamical Relay node placement solution for MANETs," Computer Communications, vol. 114, pp. 36-50, 2017.

[13] N. Papanna, A. R. M. Reddy, and M. Seetha, "EELAM: Energy efficient lifetime aware multicast route selection for mobile ad hoc networks," Applied Computing and Informatics, 2017.

[14] A. Amuthan, N. Sreenath, P. Boobalan, and K. Muthuraj, "Dynamic multi-stage tandem queue modeling-based congestion adaptive routing for MANET," Alexandria Engineering Journal, vol. 57, no. 3, pp. 1467-1473, 2018.

[15] H. A. Ali, M. F. Areed, and D. I. Elewely, "An on-demand power and load-aware multi-path nodedisjoint source routing scheme implementation using NS-2 for mobile ad-hoc networks," Simulation Modelling Practice and Theory, vol. 80, pp. 50-65, 2018.

[16] S. Hao, H. Zhang, and M. Song, "A Stable and Energy-Efficient Routing Algorithm Based on Learning Automata Theory for MANET," Journal of Communications and Information Networks, vol. 3, no. 2, pp. 52-66, Dec. 2018. 